\title{
Accelerator Information on SUSY and its Implications for Dark Matter
}

\author{
Rohini M. Godbole ${ }^{\mathrm{a}}$, Centre for Theoretical Studies, Indian Institute of Science, \\ Bangalore, 560012, India
}

\begin{abstract}
In this short contribution I want to discuss one particular candidate for the Dark Matter (DM) in the Universe, viz., the lightest supersymmetric particle (LSP). I discuss, very briefly, the motivation for Supersymmetry as well as the LSP as DM candidate. Then I summarise the current accelerator bounds on its mass and couplings and end by pointing out the implications of these limits for the experiments which search 'directly' for the DM.
\end{abstract}

Key words. Dark matter-supersymmetry.

\section{Motivation for supersymmetry (SUSY) in the standard model (SM) of particle physics}

Particle Physics at present, boasts of a description of the fundamental particles and iteractions among them, viz. the Standard Model (SM) which can describe accurately every single piece of available experimental information. Every prediction of the SM has been tested to a remarkable accuracy (de Boer et al. 1996). In spite of this phenomenal success, particle physicists are still not ready to accept the SM as the final theory, due to its various theoretical problems. A fundamental scalar (Higgs) is essential to the SM to describe the spontaneous breakdown of the Electroweak (EW) symmetry. The mass of such a scalar (or alternatively the scale of the EW symmetry breakdown) is not protected from receiving large radiative corrections in case the theory contains a mass scale other than the EW scale. Supersymmetry (a symmetry which connects bosons to fermions) cures this basic problem of the SM in a very elegant way at the cost of doubling the particle spectrum. It predicts supersymmetric partners for every known particle. None of these 'super' particles (sparticles) have been seen experimentally so far. In spite of that, a large number of particle physicists take the idea of SUSY seriously for the following reasons:

1. The measurements of coupling strengths for Weak, Electromagnetic and Strong interactions in the high precision experiments at currently available energies are consistent with the hypothesis of unification of all the three at some high energy scale, only if these so called Grand Unified Theories are Supersymmetric.

2. SUSY theories demand existence of at least one light Higgs scalar with a mass $M_{H}<130-150 \mathrm{GeV}$. The precision measurements from LEP indicate (though they don't yet force it upon us) the presence of such a light Higgs and imply that

\footnotetext{
${ }^{\mathrm{a}}$ On leave of absence from Dept. of Physics, University of Bombay, Mumbai, India.
} 
(de Boer et al. 1996)

$$
M_{H}=141_{-77}^{+141} \mathrm{GeV} \text {. }
$$

3. In almost all the attempts to unify gravity with the other three fundamental interactions SUSY almost always appears 'naturally'. The only successful theory to provide a finite theory of gravitation, viz., String Theory includes SUSY.

All this has led particle physicists to believe that the eventual 'final' description of particles and interactions among them will involve SUSY.

\section{LSP as candidate for DM}

There are people in the audience here who are much better qualified than I am to discuss whether cosmological observations imply the existence of Dark Matter (DM) and whether it is Baryonic or Nonbaryonic. Hence I will not discuss these issues here at all. I only wish to point out that in case we know that we have nonbaryonic DM, SUSY has a natural candidate. It is the Lightest Supersymmetric Particle (LSP).

In most conservative SUSY models there exists a conserved quantum number called R-parity defined by $R_{p}=(-1)^{3 B+L+S}$, where $B, L$, and $S$ describe the Baryon number, Lepton number and Spin of the particle. This has value $(-1) 1$ for all (s) particles. $R_{p}$ conservation makes the LSP absolutely stable. Absence of exotic nuclei and overall charge neutrality of the universe on large scales, tell us that such a stable object, if it exists, has to be neutral and cannot have strong interactions. SUSY theories have two such candidates for LSP (1) the SUSY partner of neutrino called sneutrino $(\widetilde{v})(2)$ the lightest neutralino $\left(\tilde{\chi}_{0}\right)$ which is a linear combination of the spartners of the neutral Higgs bosons (SUSY requires more than one of them) called Higgsinos (denoted by $\widetilde{H}_{1}, \widetilde{H}_{2}$ ) and those of the neutral EW gauge bosons $\gamma, \mathrm{Z}$ or alternatively of the $\mathrm{U}(\mathrm{l})$ and $\mathrm{SU}(2)$ gauge bosons $B, \mathrm{~W}_{3}$ (denoted by $\widetilde{B}, \widetilde{W}_{3}$ ). It is to be noted here that both these weakly interacting particles have to exist in SUSY theories. SUSY, coupled with current experimental information, also tells us that they are massive. However, the actual masses and (in the case of LSP) couplings of this Weakly Interacting Massive Particle (WIMP) depend upon the parameters of the SUSY model.

Before we discuss the dependence of the relic density of these WIMPS on the SUSY model parameters, let me briefly discuss the order of magnitude estimate of the annihilation cross-section that a good candidate for DM (say $\chi$ ) must have (Jungman et al. 1996). At temperatures $T$ much higher than $m_{x}$, the equilibrium density of the particles will be $n_{\chi}^{e q} \propto T^{3}$ and for temperatures much below $m_{x}$, the density $n_{x}^{\text {eq }} \propto \exp \left(-m_{x} / T\right)$. So if the expansion of the universe always maintained thermal equilibrium, the density of any WIMP would be very small. However, that is not the whole story. At high temperatures $\left(T \gg \mathrm{m}_{\chi}\right)$ the $\chi^{\mathrm{S}}$ are indeed in thermal equilibrium with $\chi \chi \leftrightarrow f \bar{f}, V^{+} V^{-}$, where $f, V$ denote the fermions and bosons into which the $\chi$ can decay. In this case the number density of $\chi$ would be given by the equilibrium density as a function of $m_{x} / T$ which increases with time; this is shown in Fig. 1, taken from (Kamionkowski 1996). However, as the temperature falls below $m_{x}$ the number density of these objects falls exponentially and hence the rate of annihilation $\Gamma=\left\langle\sigma_{\mathrm{A}} v\right\rangle n_{\chi}$ (here $\sigma_{\mathrm{A}}$ is the annihilation cross-section and $v$ the relative 


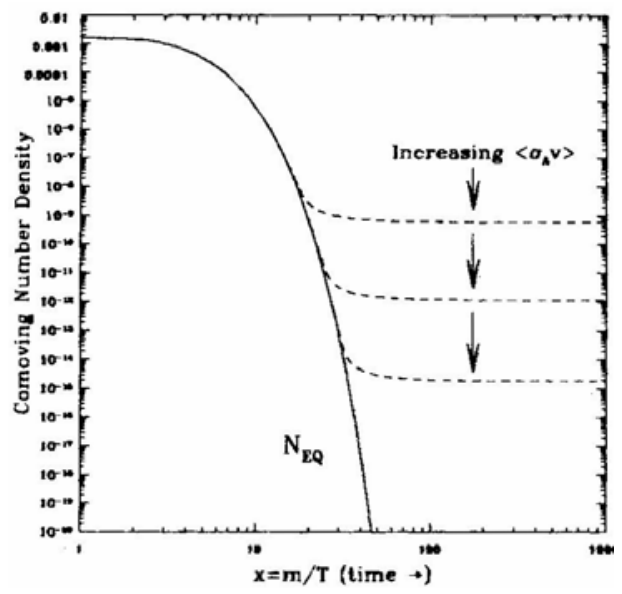

Figure 1. Comoving number density of WIMPS in the early universe. Dashed curves are the actual abundance and the solid curve is the equilibrium number density.

velocity) falls below the rate of expansion of the Universe given by the Hubble constant. At this point the $\chi \mathrm{s}$ cease to annihilate and a relic abundance can result. As can be seen from the figure the relic abundance depends on the value of $\left\langle\sigma_{\mathrm{A}} v\right\rangle$ and decreases with its increasing value as the species then remains in equilibrium till longer. An approximate solution to the Boltzman equation yields an estimate for the cosmological abundance of these WIMPs given by

$$
\Omega_{\chi} h^{2}=\frac{m_{\chi} n_{\chi}}{\rho_{c}} \simeq \frac{3 \times 10^{-27} \mathrm{~cm}^{3} \mathrm{sec}^{-1}}{\sigma_{A} v}
$$

where $h$ is the Hubble constant in units of $100 \mathrm{~km} \mathrm{~s}^{-1} \mathrm{Mpc}^{-1}$. This result, to a first approximation, is independent of the mass of the WIMP. Assuming that $v$ is a reasonable fraction of $c$, this tells us that, to get $\Omega_{\chi} h^{2} \sim \mathcal{O}(1)$, we must have $\sigma_{A} \sim 10^{-9} \mathrm{GeV}^{-2}$. As it happens, for the LSP,

$$
\sigma_{A}^{\mathrm{LSP}} \simeq \frac{\alpha_{\mathrm{em}}^{2}}{m_{\mathrm{weak}}^{2}} ;
$$

where $\alpha_{\mathrm{em}}$ is the fine structure constant and $m_{\mathrm{weak}}$ is the scale for EW interactions, viz., $\sim 200 \mathrm{GeV}$. This results in

$$
\sigma_{A}^{\mathrm{LSP}} \simeq 10^{-8} \mathrm{GeV}^{-2}
$$

Thus it proves that the LSP is a very natural candidate for DM.

The catch, however, is that the actual relic abundance of the LSP depends on the parameters of the model, which decides both the mass and couplings of the LSP. The most popularly used one is the Minimal Supersymmetric Standard Model (MSSM). A whole range of predictions for $\Omega_{\chi}$ is possible, depending upon the chosen point in the parameter space of the MSSM.

To discuss the general features of this parameter dependence of the relic density, let us first enumerate the parameters. A general LSP state is a linear combination,

$$
\tilde{\chi}_{0}=a_{1} \tilde{B}+a_{2} \tilde{W}_{3}+a_{3} \tilde{H}_{1}+a_{4} \tilde{H}_{2} .
$$


The $a_{i}$ 's depend on the SUSY parameters:

1. The $\mathrm{U}(1), \mathrm{SU}(2)$ and $\mathrm{SU}(3)$ gaugino masses, $M_{1}, M_{2}, M_{3}$ (gauginos are spartners of gauge bosons).

2. The Higgsino mass term, $\mu$.

3. Tan $\beta=v_{2} / v_{1}$, where $v_{1}, v_{2}$ are the vacuum expectation values acquired by the Higgs fields, which break EW symmetry.

The parameters $M_{i}$, are a measure of SUSY breaking. Again, assuming unification, the number of parameters can be reduced to three: $M_{2}, \mu$ and $\tan \beta$. Unification implies $M_{1} \simeq 0.5 M_{2}$ at the weak scale of $\sim 200 \mathrm{GeV}$. The couplings and masses of the LSP are decided by these three parameters. They can be classified broadly as

- $|\mu| \gtrsim M_{2}$ : This gives the LSP to be mostly a photino (spartner of photon) for $M_{1} \ll M z$ For $M_{1}>M z$, it is mostly a bino $(\tilde{B})$ with $m_{\tilde{\chi} 0} \sim M z$.

- $|\mu| \simeq M_{2}$ : This gives an LSP which is really a mixture, i.e., all $a_{i}$ 's are comparable.

- $|\mu|<M_{2}$ : This gives an LSP which is mostly a Higgsino, i.e., $a_{1}, a_{2} \ll a_{3}, a_{4}$; $m_{\tilde{\chi}^{0}} \simeq \mu$.

Fig. 2 taken from (Drees \& Nojiri 1993) shows contours of varying $\Omega_{\chi} h^{2}$ in the $M\left(M_{3}\right), \tan \beta$ plane. The relic density depends on $M, \tan \beta, \mu$, as well as the common scalar mass, $m$, of the spartners of all fermions. Initially, it was thought (Greist \& Seckel 1991; Mizuta \& Yamaguchi 1993; Edsjõ \& Gondolo 1997) that a Higgsinolike LSP will have to be very heavy $\left(m_{\tilde{\chi}_{0}}>0.5 \mathrm{TeV}\right)$ as otherwise $\sigma_{A}$ is too large. However, it was recently shown (Drees et al. 1997) that even a light Higgsino can be a viable candidate for DM due to the loop corrections to couplings and masses of the LSP. This is good news as the MSSM predicts measurable signals at the current colliders, LEP, LEP-II and the TeVatron for the range of model parameters for which LSP is a viable dark matter candidate. This is the subject of discussion in the next section.

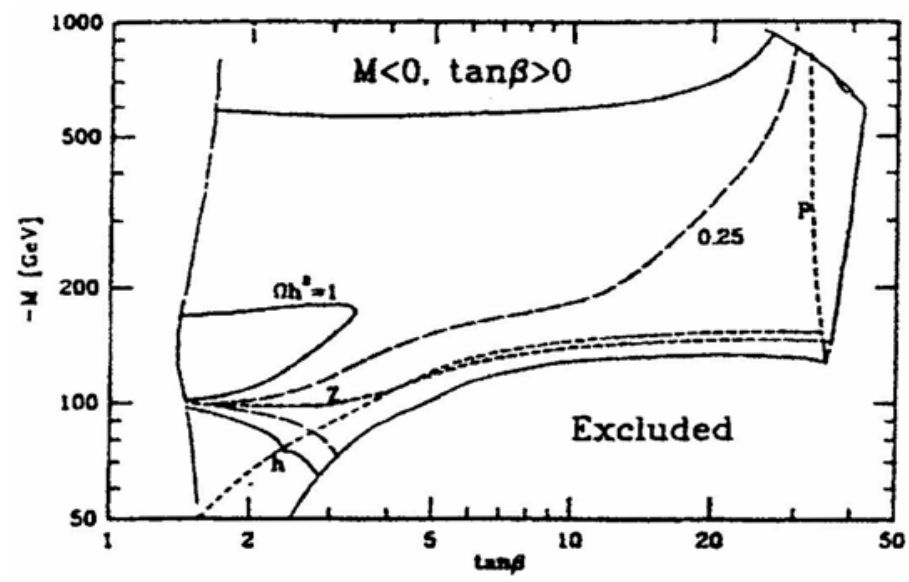

Figure 2. Example of contours of constant $\Omega_{\chi} h^{2}=1$ (solid lines) $=0.25$ (long dashed lines) in the $M, \tan \beta$ plane for $m_{t}=140 \mathrm{GeV}$ and $m=250 \mathrm{GeV}$. The region outside the outer dotted lines is excluded by various experimental and theoretical constraints other than the DM relic density. 


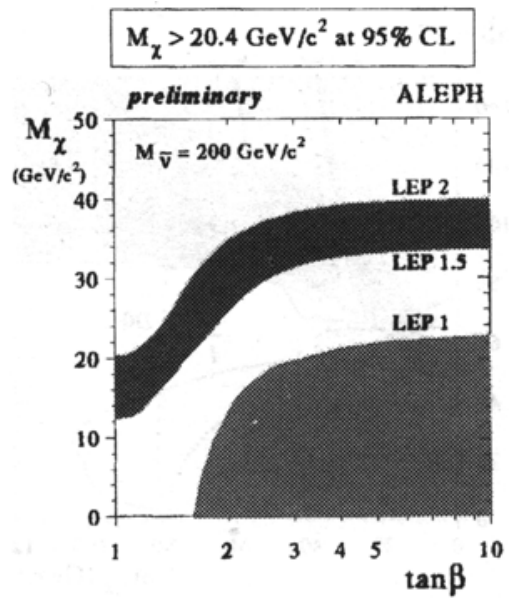

Figure 3. Experimental lower bounds on the neutralino mass: note that neither LEP1 nor LEP1.5 data impose a nonzero lower bound, though their combination does.

\section{Current accelerator constraints on LSP and its implications for DM}

At the current accelerators, the most stringent limits on the LSP mass/couplings of neutralinos and charginos (charged counterparts of neutralinos) come from their nonobservation at the experiments at LEP $\left(e^{+} e^{-}\right.$collisions on the $\mathrm{Z}$ pole $)$ and LEP 1.5, LEP160 and LEP2 with collision energies 130, 160, and $172 \mathrm{GeV}$ respectively. These can be produced in $e^{+} e^{-} \rightarrow \tilde{\chi}_{0} \tilde{\chi}_{0}, e^{+} e^{-} \rightarrow, \tilde{\chi}_{0} \tilde{\chi}_{1}$ and $e^{+} e^{-} \rightarrow \tilde{\chi}_{i}^{+} \tilde{\chi}_{j}^{-}(i, j=1,2)$. One of the results of this analysis is that one can put an absolute bound $\mathrm{m}_{\tilde{\chi}_{0}}>20.4 \mathrm{GeV}$ for the MSSM, independent of $\tan \beta$. This is shown in Fig. 3, taken from (Ellis et al. 1996). (The limit now has been raised to $27.4 \mathrm{GeV}$ in the more recent analysis with the LEP 2 data (Ellis et al. 1997)).

This analysis also imposes all the other existing constraints from continued nonobservation of the superparticles at current $\bar{p} p$ and lower energy $e^{+} e^{-}$experiments. Fig. 4, taken from Ellis et al. (1996) shows the region in $M_{2}$ VS. $m$ plane (indicated as $\mathrm{m}_{1 / 2}$ and $m_{0}$ respectively in the figure) which is allowed by current experiments. Also shown in the figure are regions (light-shaded for some experimentally-allowed value of $\mu<0$ and dark-shaded for $\mu$ determined by dynamical EW symmetry breaking) where the LSP can be a viable dark matter candidate and provide $0.1<\Omega_{x} h^{2}<0.3$. As one can see, there exists a large region in the allowed parameter space where the LSP can be a viable dark matter candidate.

\section{Implications of the accelerator constraints for DM detection}

The search for SUSY at accelerator experiments indeed provides an indirect probe of the DM. The constraints on the SUSY parameters put by these experiments also have implications for the detection experiments for these DM candidates. The limit on $m_{\tilde{\chi} 0}$ mentioned above implies that these detection experiments need not look for $m_{\tilde{\chi} 0}<20 \mathrm{GeV}$ in case $\tilde{\chi}_{0}$ is the DM candidate.

There are two types of these DM detection experiments: 


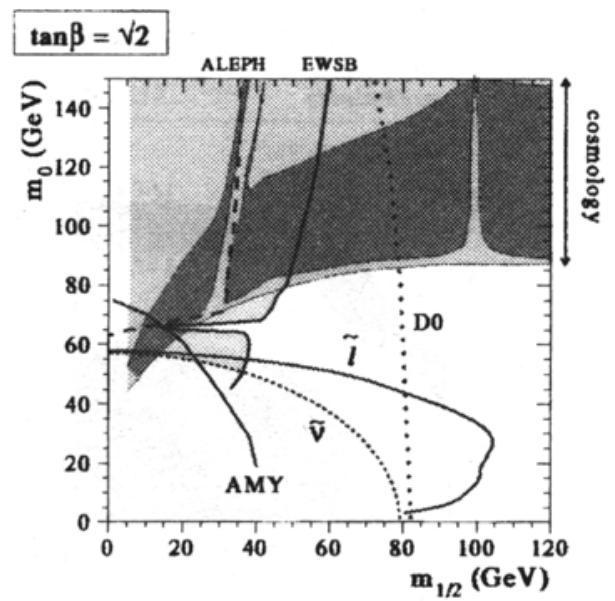

Figure 4. The domain of the $\left(m_{1 / 2}, m_{0}\right)$ plane for $\mu<0$ and $\tan \beta=\sqrt{2}$ that is excluded by ALEPH chargino and neutralino searches (long dashed line), by the $Z^{0}$ limit on $m_{\tilde{v}}$ (short dashed line), by the LEP limits on slepton production (solid line), by single photon measurements (grey line), and by the D0 limit on the gluino mass (dotted line).

\section{Direct detection}

These experiments look for interactions of $\chi$ with nuclei by measuring the recoil energy of the nucleus. Germanium is one of the commonly used detectors. The predicted event rate depends on (1) the nucleus and (2) $m_{x}$ and (3) the nature of the coupling of $\chi$ with matter. A scalar coupling would yield larger rates. For the allowed range of MSSM parameters, which provide appreciable relic density, one predicts between $10^{-4}-10$ events per $\mathrm{kg}$ per day. The sensitivity of current detectors is about 10 events per kg per day. These experiments, when combined with LEP limits, have already ruled out a sneutrino as a DM candidate (Quenby et al. 1995).

\section{Indirect detection}

Here one looks for energetic neutrinos which are emitted due to annihilation of $\chi$ inside the Sun. For this, the currently available neutrino detectors are suitable. The expected flux varies between $10^{-6}-1$ per $\mathrm{m}^{2} \mathrm{~s}^{-1}$. Rates are higher for axial vector coupling. Since, for MSSM, the predictions vary over a very wide range, any pointers provided by current experiments are very helpful.

I wish to emphasise here the symbiotic relationship between these low energy experiments and the accelerator search experiments. For example, an indirect detection of WIMP could tell us immediately that SUSY is $R_{p}$ symmetric. So, even though the experiments at LEP2 and/or large hadron collider (LHC) should discover SUSY, the DM detection experiments still will not be any less important. On the other hand, if the sparticles are really heavy (beyond the reach of LHC), then these experiments might be our only window to the physics beyond the electroweak scale. 


\section{References}

de Boer, W., Dabelstein, A., Hollik, W., Mosle, W., Schwickerath, U, 1996, (IEKPKA/96-08, hep-ph/9609209). See also Z. Phys. C75 (1997) 627.

Drees, M., Nojiri, M. M. 1993, Phys. Rev., D47, 376.

Drees, M., Nojiri, M. M., Roy, D. P., Yamada, Y. 1997, (KEK-TH 505, hep-ph/9701219); Drees, M. (hep-ph/9701318), Talk presented at 2nd International RESCU Symposium on Dark Matter and its Direct Detection, Tokyo, Japan.

Ellis, J., Falk, T., Olive, K. A., Schmitt, M. 1996, (hep-ph/9610410), Talk presented by J. Ellis at Workshop on Identification of the Dark Matter, Sheffield, U.K.

Ellis, J., Falk, T., Olive, K. A., Schmitt, M. 1997 (hep-ph/9705444); Phys. Lett., B413, 355.

Edsjõ, J., Gondolo, P. 1997, (hep-ph/9704361); Phys. Rev., D56, 1879.

Greist, K., Seckel, M. 1991, Phys. Rev., D43, 3191

Jungman, G., Kamionkowski, M., Greist, K. 1996, Phys. Rep., 267, 195.

Kamionkowski, M. 1996, (hep-ph/9609531), Talk presented at VIIIth Recontres de Blois, Neutrinos, Dark Matter and the Universe, Blois, France.

Mizuta, S., Yamaguchi, M. 1993, Phys. Lett., B298, 120

The currently best published WIMP detection limits are: for direct detection: J. J. Quenby et al. 1995, Phys. Lett., B357, 70; for indirect detection: Baskan collaboration, as presented at TAUP95, Valencia, Spain, September 1995. 